\title{
On advances and challenges in biocatalysis
}

\author{
In this Editorial, we discuss recent advances and challenges in the field of biocatalysis and introduce some relevant
} work you will find in this issue of Nature Catalysis.

W hile attending the recent Gordon Research Conference on

biocatalysis frontiers, the increasing pace of developments in this field was certainly noted. The conference spanned a broad range of disciplines in biocatalysis research and was highly topical with many unpublished discoveries presented in the talks and posters. In a recent report, the global enzymes market was valued at over US $\$ 7$ billion in 2017, and is projected to reach over US $\$ 10$ billion by 2024 (ref. $^{1}$ ). From this viewpoint, the high percentage of industrial participants (circa $30 \%$ of around 200 conference attendees) is not unexpected. A possible driver to further increase the market share of biocatalysis is the expansion of the biocatalytic toolbox. Nowadays, chemistry that was exclusively reserved to organic chemists, such as carbonsilicon and carbon-boron bond formation, can be mediated by metalloenzymes that have been engineered by directed evolution of a natural protein ${ }^{2,3}$. A promising complementary strategy is to combine the selectivity provided by a protein scaffold with the broad scope of synthetic catalysts. However, recycling of the single components of such artificial metalloenzymes is challenging. In an Article in this issue of Nature Catalysis, Anne-Kathrin Duhme-Klair, Keith S. Wilson and colleagues report a methodology for controlling assembly and disassembly of artificial metalloenzymes, which can, for example, be used to replace a poisoned synthetic catalyst in the protein scaffold.

Biocatalysis is very appealing for industry because it allows in principle the synthesis of products not accessible by chemical synthesis, use of alternative raw materials, lower operating costs, low fixed-cost infrastructure and improved eco-efficiency. Enzyme cascades - dedicated with a special talk session in the conference programme - are particularly interesting, because purification and isolation of intermediates is omitted, which leads to reductions in operating time, production costs and waste. Recent successful examples utilizing cascades are the production of opioids in yeast utilizing 21-23 enzymes from plants ${ }^{4}$, and the production of primary flavour determinants in hopped beer ${ }^{5}$. In an Article in this issue, Sang Yup Lee and colleagues report the secretory production of free haem - a complex molecule and powerful iron-supplying agent used in the healthcare and food supplements industries - by metabolically engineered Escherichia coli strains. This constitutes a very powerful strategy as the chemical synthesis has proven to be low yielding and time consuming. In another Article in this issue, Itamar Willner and colleagues demonstrate that the activity and stability of enzyme cascades can be distinctively improved for biotechnological applications by operating them in metalorganic framework nanoparticles.

The conference talks highlighted that machine learning and automation in data analysis, library design and gene synthesis today facilitates the tailoring of biocatalysts for industrial processes in very short periods. Structural and dynamic information contribute to reduced timeframes, being extremely valuable for the design of smart libraries for screening. Another conference session discussed the advances in cryo-electron microscopy, X-ray freeelectron laser (XFEL) crystallography and biodynamic nuclear magnetic resonance. Serial femtosecond nanocrystallography using free-electron lasers is a promising new technique. For example, it has allowed structural changes and the site of $\mathrm{O}=\mathrm{O}$ bond formation in photosystem II to be studied at room temperature, without structure-affecting radiation damage . $^{6}$ It is anticipated that this technique will also allow the determination of molecular movies of biomolecules and enzymes during catalysis. This could be made possible by further improvements in data collection and analysis, and by new XFEL facilities such as the European XFEL in Hamburg (Germany), which was opened in 2017 with a construction cost amounting to $€ 1.25$ billion and a length of $3.4 \mathrm{~km}$. It provides an XFEL with a much higher intensity compared with conventional synchrotron light sources, allowing shorter exposure time, higher resolution and a greater number of snapshots (https://www.xfel.eu).

\section{Despite the breathtaking}

developments in and around the field of biocatalysis, the conference attendees concluded that a persistent, unresolved challenge is making biocatalysis more predictive.
Despite the breathtaking developments in and around the field of biocatalysis, the conference attendees concluded that a persistent, unresolved challenge is making biocatalysis more predictive. It is as yet impossible to accurately predict all enzyme parameters from a sequence. Succeeding in this undertaking would revolutionize biocatalysis, making the way in which we conduct science in this research area and related fields much more constructive. For example, biocatalysis research undoubtedly benefits from the ever-increasing amount of available sequencing data. However, accurately annotating the function of biological macromolecules based on their sequences is not trivial. As an illustrative example, a recently designed minimal bacterial genome (derived from Mycoplasma mycoides) that constituted just 473 genes contained 149 genes with unknown biological functions ${ }^{7}$. Many scientists would clearly benefit from improvements in this area, as correct annotation of enzyme functions could be useful in assembling suitable metabolic engineering pathways, aid the investigation of biosynthetic pathways, and provide a broadened set of enzyme classes and subgroups for biocatalytic synthesis and enzyme engineering. In contrast, a wrongly annotated function can, for example, mask sought-after enzymes and unknown enzyme classes.

Accurately predicting protein parameters, such as three-dimensional structure, protein dynamics, protein electrostatics and catalytic parameters, would help scientists to better direct their efforts, reducing the necessary experimental work. For example, computational optimization of electric fields was recently successfully used to reduce the necessary experimental effort to optimize the performance of a synthetic enzyme $^{8}$. In a Perspective in this issue, Teresa Head-Gordon and colleagues discuss recent progress and future directions in the computational optimization of electric fields for better design of chemical and biological catalytic systems. To date, the efficiency of de novo-designed biocatalysts is still distinctly lower compared with naturally evolved enzymes, indicating that the predictive capacity 
of the employed theoretical models has room for improvement.

In summary, the biocatalysis field is rapidly moving forward with the introduction of abiological reactions and ongoing advances in enzyme engineering. In addition, emerging structural techniques are anticipated to give new insights into enzyme catalysis. However, an improved predictive capacity of theoretical models is desired to make biocatalysis engineering and research more constructive.

Published online: 12 September 2018

https://doi.org/10.1038/s41929-018-0157-7

References

1. Srivastava, A. \& Srivastava, M. Enzymes Market-Global Opportunity Analysis and Industry Forecast, 2017-2024 (2018); https://www.alliedmarketresearch com/enzymes-market 2. Kan, S. J., Lewis, R. D., Chen, K. \& Arnold, F. H. Science 354, 1048-1051 (2016).

3. Kan, S. J., Huang, X., Gumulya, Y., Chen, K. \& Arnold, F. H. Nature 552, 132-136 (2017).

4. Galanie, S., Thodey, K., Trenchard, I. J., Interrante, M. F. \& Smolke, C. D. Science 349, 1095-1100 (2015).

5. Denby, C. M. et al. Nat. Commun. 9, 965 (2018).

6. Suga, M. et al. Nature 543, 131-135 (2017).

7. Hutchison, C. A. et al. Science 351, aad6253 (2016).

8. Vaissier, V., Sharma, S. C., Schaettle, K., Zhang, T. \& Head-Gordon, T. ACS Catal. 8, 219-227 (2017). 